\title{
Development of advanced seed laser modules for lidar and spectroscopy applications
}

\author{
Narasimha S. Prasad ${ }^{1}$, Alex Rosiewicz ${ }^{2}$, Steven M. Coleman ${ }^{2}$ \\ ${ }^{1}$ NASA Langley Research Center, Laser Remote Sensing Branch, Hampton, VA 23681; \\ ${ }^{2}$ Gooch \& Housego Boston (EM4 Inc.), 7 Oak Park Drive, Bedford, MA 01775
}

\begin{abstract}
We report on recent progress made in the development of highly compact, single mode, distributed feedback laser (DFB) seed laser modules with integrated drive electronics for lidar and spectroscopy applications from space based platforms. One of the intended application of this technology is in the NASA's Active Sensing of CO2 Emissions over Nights, Days, and Seasons (ASCENDS) mission. NASA Langley Research Center (LaRC) is working on a prototype laser based spectroscopy system for simultaneous measurement of $\mathrm{CO}_{2}$ and $\mathrm{O}_{2}$ for planned Active Sensing of $\mathrm{CO}_{2}$ Emissions over Nights, Days, and Seasons (ASCENDS) mission application. For this purpose, $1571 \mathrm{~nm}$ spectral band for $\mathrm{CO}_{2}$ sensing and $1262 \mathrm{~nm}$ spectral band for oxygen sensing have been selected. In this paper, we discuss recent progress made in the development of single mode, compact and stable, seed laser technologies for $\mathrm{CO}_{2}$ and $\mathrm{O}_{2}$ transmitters with focus on linewidth and noise measurements. The $1571 \mathrm{~nm}$ and $1262 \mathrm{~nm}$ DFB laser modules with integrated drive electronics have advanced current and temperature drivers built into them. A combination of temperature and current tuning allows coarse and fine adjustment of the diode wavelengths. The current tuning was demonstrated at a rate of $\sim 0.7 \mathrm{pm} / \mathrm{mV}$ over a working range of $\sim 1 \mathrm{~V}$ for a total of $0.7 \mathrm{~nm}$. Also, temperature tuning at a rate of $\sim 2 \mathrm{pm} / \mathrm{mV}$ over a working range of $\sim 1 \mathrm{~V}$ for a total wavelength range of $\sim 2 \mathrm{~nm}$ was demonstrated. The current tuning was performed at a rate of up to $200 \mathrm{kHz}$ allowing rapid adjustment and dithering of the laser frequency. Furthermore, the best performance of laser linewidth observed was $\sim 11 \mathrm{kHz}$ with frequency stability $<10 \mathrm{MHz}$ over 1 hour period. The microcooler arrangement embedded inside these modules has provided significant reduction in power consumption. The electronics has been designed, prototyped and tested using space-qualified components within a hermetically sealed package of volume less than $2 "$ x 2" x $0.5 "$.
\end{abstract}

Keywords: ASCENDS, $\mathrm{CO}_{2}, \mathrm{O}_{2}$, DFB laser diode

\section{INTRODUCTION}

The National Research Council (NRC) Decadal Survey of Earth Science and Applications from Space has identified has identified Active Sensing of CO2 Emissions over Nights, Days, and Seasons (ASCENDS) as an important mid-term (tier II) and mid-size mission [1,2]. Figure 1 illustrates the ASCENDS mission goals. The ASCENDS mission is anticipated to deliver laser based remote sensing measurements of $\mathrm{CO}_{2}$ mixing ratios $\left(\mathrm{XCO}_{2}\right)$ day and night, at all latitudes, and during all seasons. ASCENDS includes simultaneous measurements of (a) $\mathrm{CO}_{2}$ number density tropospheric column, (b) $\mathrm{O}_{2}$ number density column that allows surface pressure measurement to derive average $\mathrm{XCO}_{2}$ column, (c) Temperature profile to obtain improved $\mathrm{CO}_{2}$ accuracy, (d) Altimetry measurements to determine surface elevation, cloud top heights, (e) carbon monoxide (CO) profile to identify combustion sources of $\mathrm{CO}_{2}$. ASCENDS is the logical extension of (orbiting Carbon Observatory (OCO) and Greenhouse gases Observing SATellite (GOSAT) missions. 
NASA Langley Research Center, in partnership with ITT, is working on a novel CW Laser Absorption Spectrometer (LAS) technique for simultaneous measurement of the $\mathrm{CO}_{2}$ column number density and the $\mathrm{O}_{2}$ column number density. Due to architectural advantages combined with reasonable spectroscopic features, the $1571 \mathrm{~nm}$ wavelength band is selected for $\mathrm{CO}_{2}$ measurements. For $\mathrm{O}_{2}$ measurements to determine surface pressure, the $12 \mathrm{xx} \mathrm{nm}$ band has been selected. The $\mathrm{O}_{2}$ column number density is used to convert the column number density to $\mathrm{CO} 2$ mixing ratio $\left(\mathrm{XCO}_{2}\right)$. An altimeter based on a pulsed laser operating around $1591 \mathrm{~nm}$ is utilized for path length measurement. Plans are underway to incorporate (a) a six channel passive radiometer based temperature profiler for constraining the $\mathrm{XCO}_{2}$ measurement, and (b) a passive $\mathrm{CO}$ measuring scheme using a gas filter correlation radiometer to separate biogenetic fluxes from biomass burning fossil fuel combustion to cover the same the $\mathrm{XCO}_{2}$ path for interpreting sources and sinks of $\mathrm{CO}_{2}$. Furthermore, an imager is being used to provide cloud clearing for soundings. Currently, NASA LaRC is collecting data from Commercial of the shelf (COTS) based airborne instruments to verify the $\mathrm{CO}_{2}$ measurement capability of the laser based LAS approach. In this context, NASA is looking into advances in instrumentation concepts for reducing size, weight, volume, and power consumption while enhancing the signal-to-noise ratio of return signals. In this paper, advancements in seed laser techniques for ASCENDS transmitters are presented in the following sections.

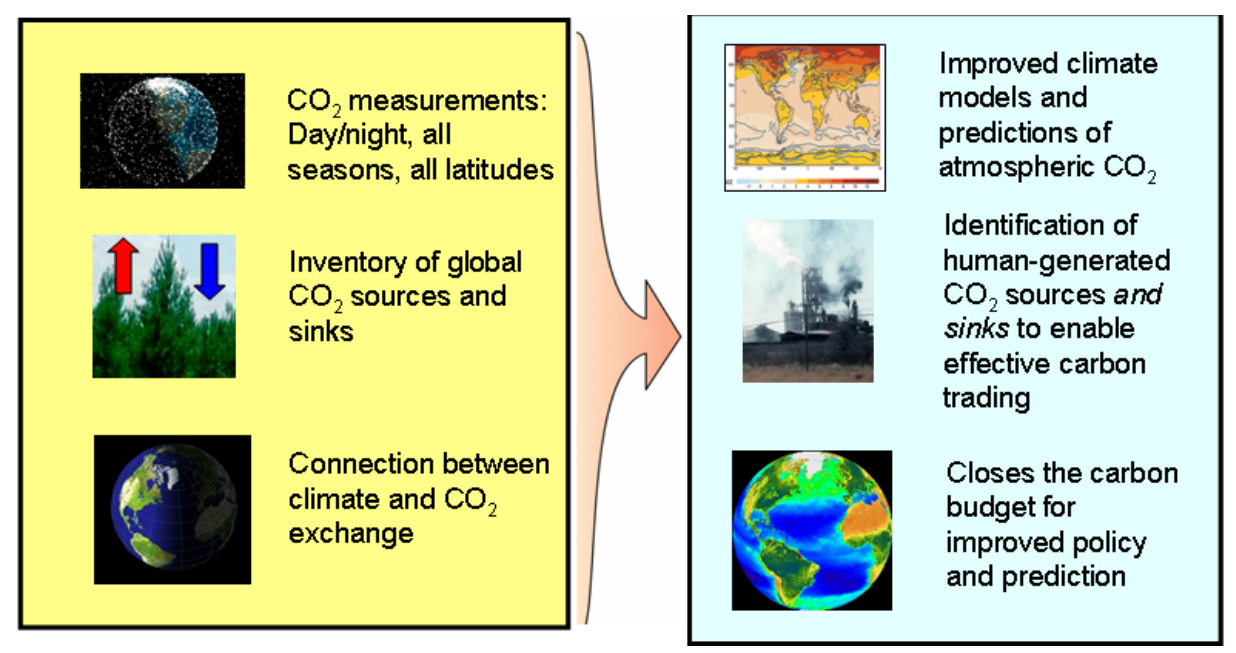

Figure 1. ASCENDS mission goals [1,2].

\section{SEED LASER DEVELOPMENT}

Advanced distributed feedback (DFB) diode-laser concepts and technologies are necessary to facilitate NASA LaRC's ongoing ASCENDS LAS transmitter to effectively satisfy space platform instrumentation requirements. Efficient, compact, robust, and reliable seed laser and fiber amplifier modules are being developed to meet operational and payload challenges. In this paper, the design features and preliminary performance characteristics of a low Size, Weight and Power (SWaP) transmitter operating at $1571 \mathrm{~nm}$ and $1262 \mathrm{~nm}$ including experimental data are presented.

The objectives of this ongoing effort are to design, build and test space qualifiable SWAP seed laser component prototypes operating in the $1571 \mathrm{~nm}$ and $1260 \mathrm{~nm}$ spectral bands. The design steps include: (a) development of high power $(\sim 100 \mathrm{~mW})$, novel, 12xx nm wavelength DFB laser chips compatible with the package and drive electronics, (b) fabricate 14-pin butterfly DFB laser packages to improve thermal efficiency and reduce component mass for $1571 \mathrm{~nm}$ and 12xx lasers, and (c) integration of laser chips and drive electronics in 14-pin butterfly package to produce compact DFB laser modules operating. In addition, techniques to integrate all associated drive and control electronics coupled to novel nano-cooler scheme in a single hermetically sealed package are being investigated. The performance objectives of the seed laser module identified are: (a) size < 1.5" x 1.5" x 0.7 ", (b) weight $<100 \mathrm{gm}$, and (c) overall power consumption $<1 \mathrm{~W}$. To accomplish these objectives, a modified-COTS $1571 \mathrm{~nm}$ DFB seed laser module, incorporating a low-noise variable laser bias current supply and low-noise variable temperature control circuit, has been developed and 
tested. A similar module operating in the $1262 \mathrm{~nm}$ spectral band is being fabricated. It is anticipated that these components will satisfy Technology Readiness Level-TRL 6 requirements for ASCENDS.

\section{BASELINE ARCHITECTURE}

Figure 2 shows the previously developed baseline design architecture module EM600 that is now being modified to incorporate the above enumerated features. The performance features of the baseline EM600 module are: (a) operational wavelength of 1.571 microns, (b) typical output power is $40 \mathrm{~mW}$, (c) spectral linewidth $\sim 300 \mathrm{kHz}$, (d) output wavelength tenability of $\pm 1.15 \mathrm{pm} / \mathrm{mV}$, (d) freq tuning via temperature tuning @ $140 \mathrm{MHz} / \mathrm{mV}$, (e) freq tuning achieved via power adjustment @ 28MHz/mV, (f) output power tuning @ 18uW/mV, (g) fiber coupled output, (h) hermetically sealed laser, (i) non-space-qualified electronics, and (j) TRL 4 [3-5].

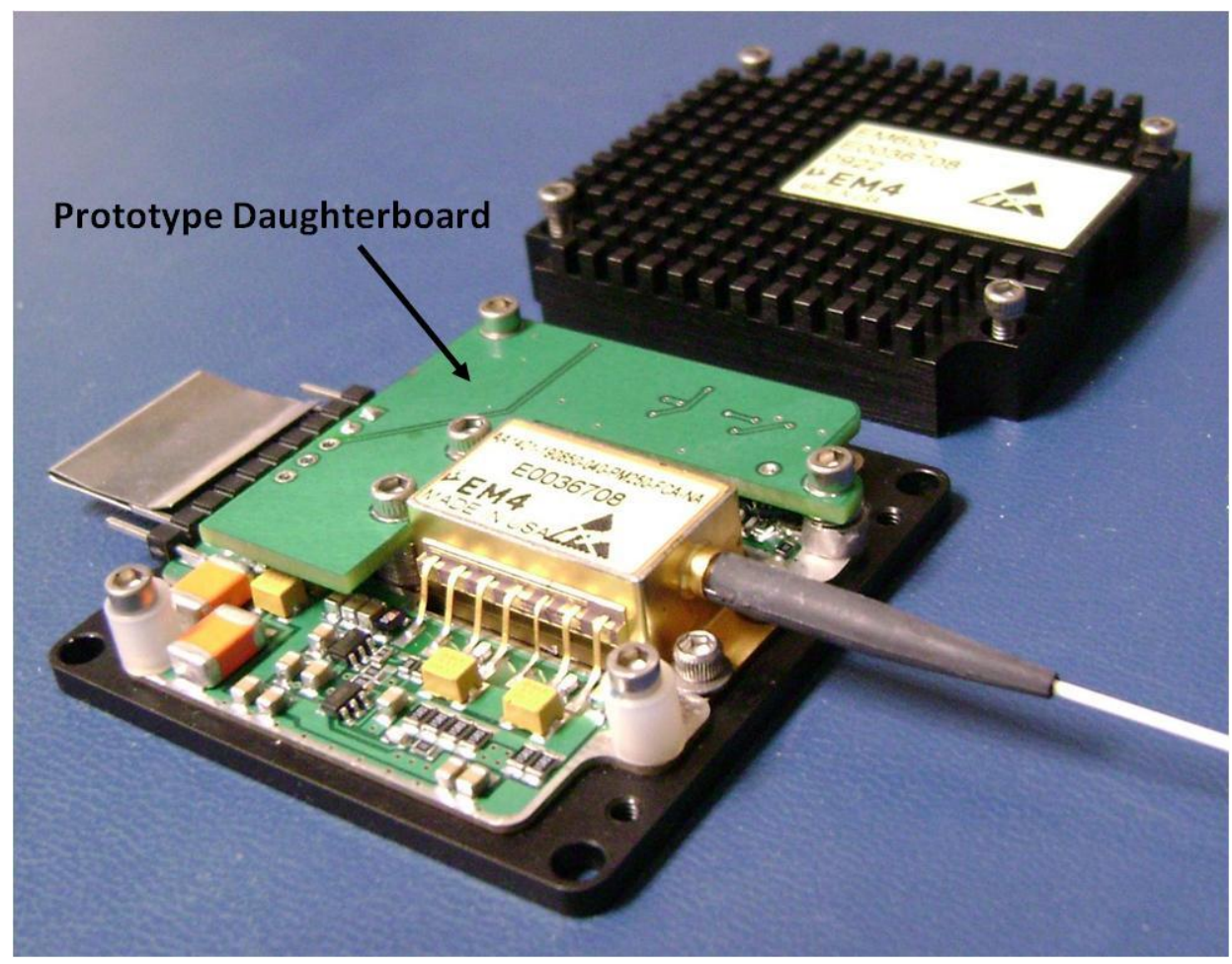

Figure 2. The EM600 laser transmitter module where size, weight and power (SWAP) have been greatly reduced by incorporating drive electronics into the same hermetic enclosure as the laser chip and coupling optics. The overall dimensions of this package is $\sim 2 " \times 2 " \times 0.5 "$.

The output power and wavelength tunability characteristics are shown in Figure 3. The variability of output power with Power Adjust (PA) and temperature tuning characteristics are shown in Figures 3a and 3b, respectively. The EM600 device is designed to run in a constant current mode with the laser current internally set for an output of $40 \mathrm{~mW}$. The output power can be decreased by applying a voltage to PA. The output power will linearly decrease with increasing voltage on PA. The PA is $50 \mathrm{Ohm}$ terminated internally. In the case of temperature tuning, the output wavelength is tuned at a rate of $+1.15 \mathrm{pm} / \mathrm{mV}$. In units of temperature, the set point tunes at a rate of $-11 \mathrm{mK} / \mathrm{mV}$. Figure 4 illustrates the frequency (wavelength) tuning and modulation chirp characteristics. Modulation of bias induces significant chirp. In our case, $80 \%$ modulation chirps laser $\sim 450 \mathrm{pm}$ at $1569.733 \mathrm{~nm}$. Figure 5 illustrates the combined tuning characteristics of EM600. Fine wavelength adjustment is accomplished by varying the drive current. As mentioned in Figure 4, the measured tuning rate is $0.2 \mathrm{pm} / \mathrm{mV}$ with a stability of $0.8 \mathrm{pm}$ over 100 seconds. The stability of the temperature tuning loop appears to be unaffected by the incorporation of the new control signal with a short term $(100 \mathrm{~s}$ average) laser frequency stability of $100 \mathrm{MHz}$. 

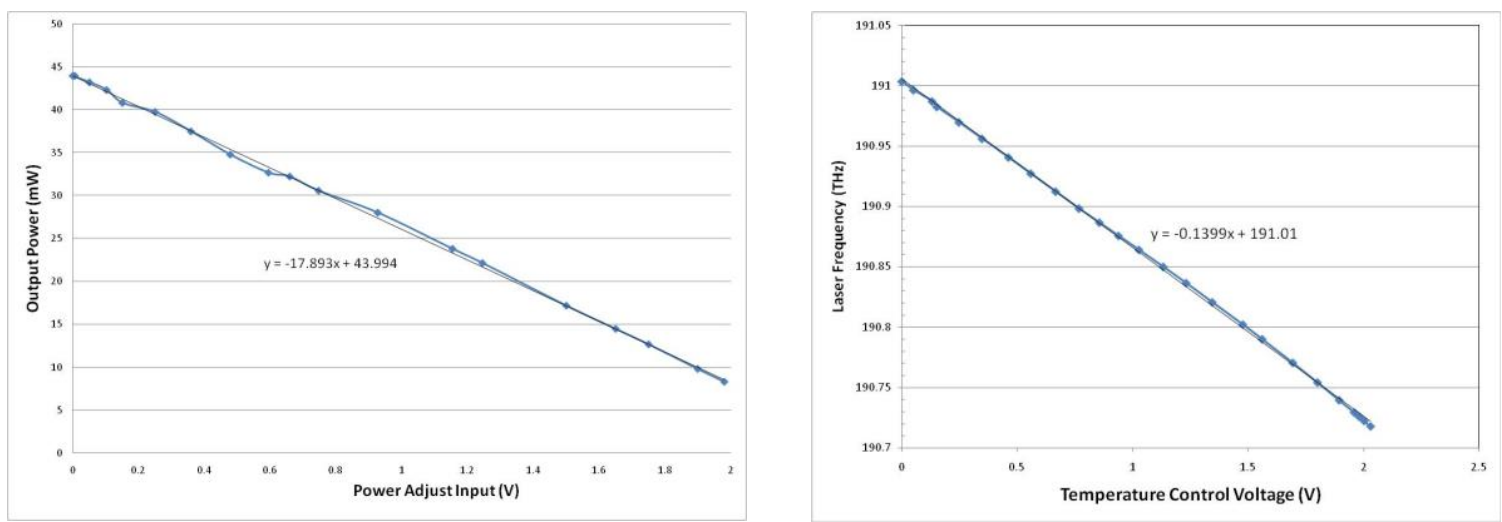

Figure 3. Operational characteristics of EM600 (a) Output power characteristics, (b) Temperature tuning characteristics.
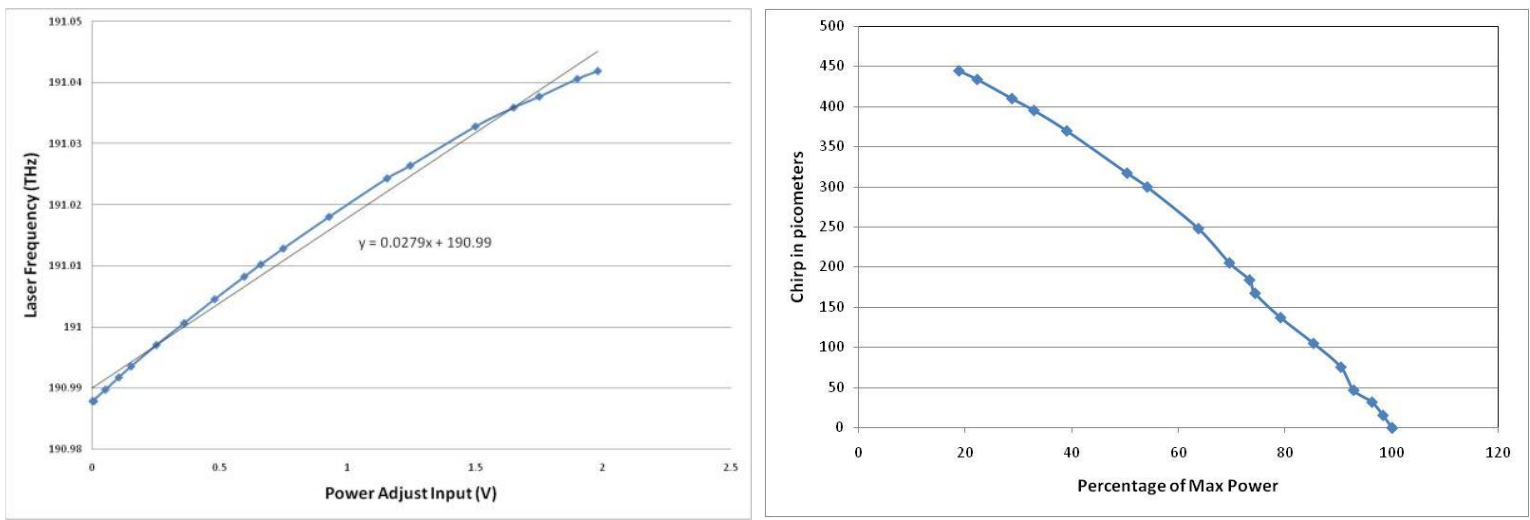

Figure 4. (a) Frequency fine tuning accomplished through power adjust control. Voltage tuning of $+0.2 \mathrm{pm} / \mathrm{mV}$ is achieved with a stability of $0.8 \mathrm{pm}$ over 100 seconds. The device tunes with opposite slope that of temperature, (b) measured modulation chirp characteristics. 


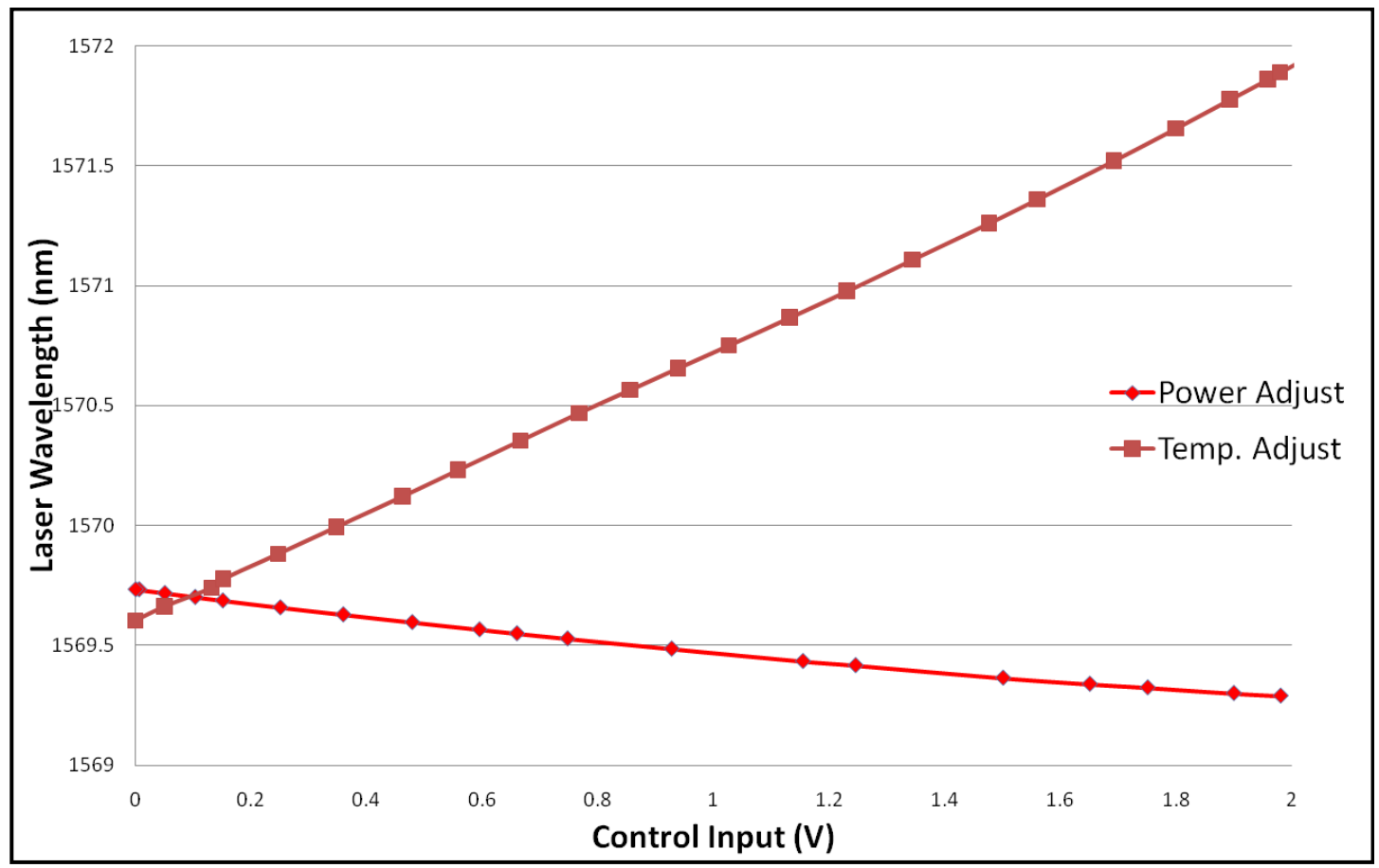

Figure 5. Combined tuning characteristics involving power adjust and temperature adjust controls.

\section{ADVANCED SEED LASER MODULE CHARACTERISTICS}

The EM650, a modified version of EM600 package for $1571 \mathrm{~nm}$ operation for improved performance characteristics has been developed and is now undergoing tests. The temperature tuning, current tuning, power stability and frequency stability of EM650 performed better than the EM600 version. Furthermore, the engineering designs for 1.26xx have been completed. The device technology will be extended to 126x.x nm wavelength band for oxygen LAS applications. Three prototype 1.571 laser diode modules were fabricated. Figure 6 shows one of the prototypes.

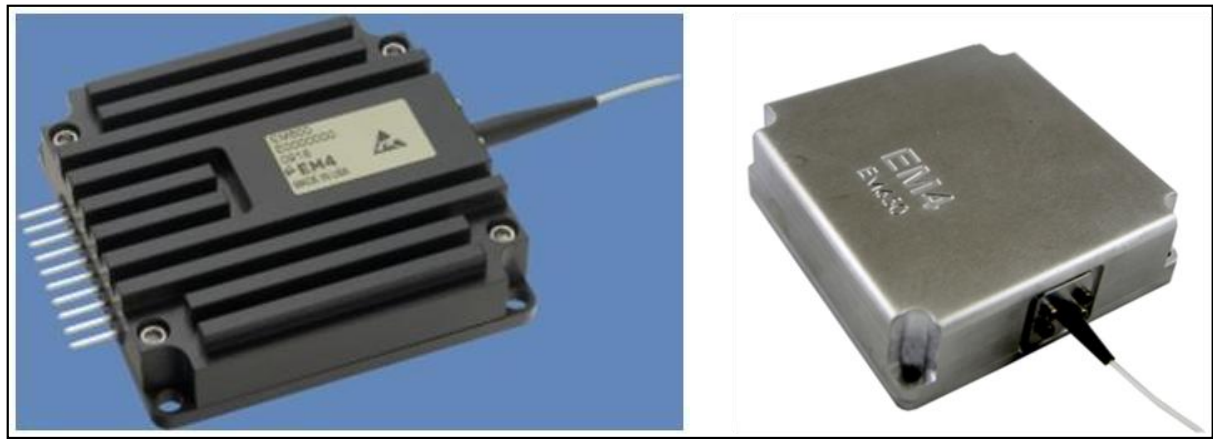

Figure 6. The COTS DFB Laser Module with Integrated Drive Electronics and Temperature Control Circuits. One of three initial production prototype EM650s.

Frequency tuning and Power Adjust characteristics were measured and they were found to be fairly linear as shown in Figure 7. Frequency jitter characteristics indicated less than $70 \mathrm{MHz}$ frequency deviation over $100 \mathrm{~s}$ interval at 25C. [4]. In the following sections, recent results on linewidth measurements are discussed. 

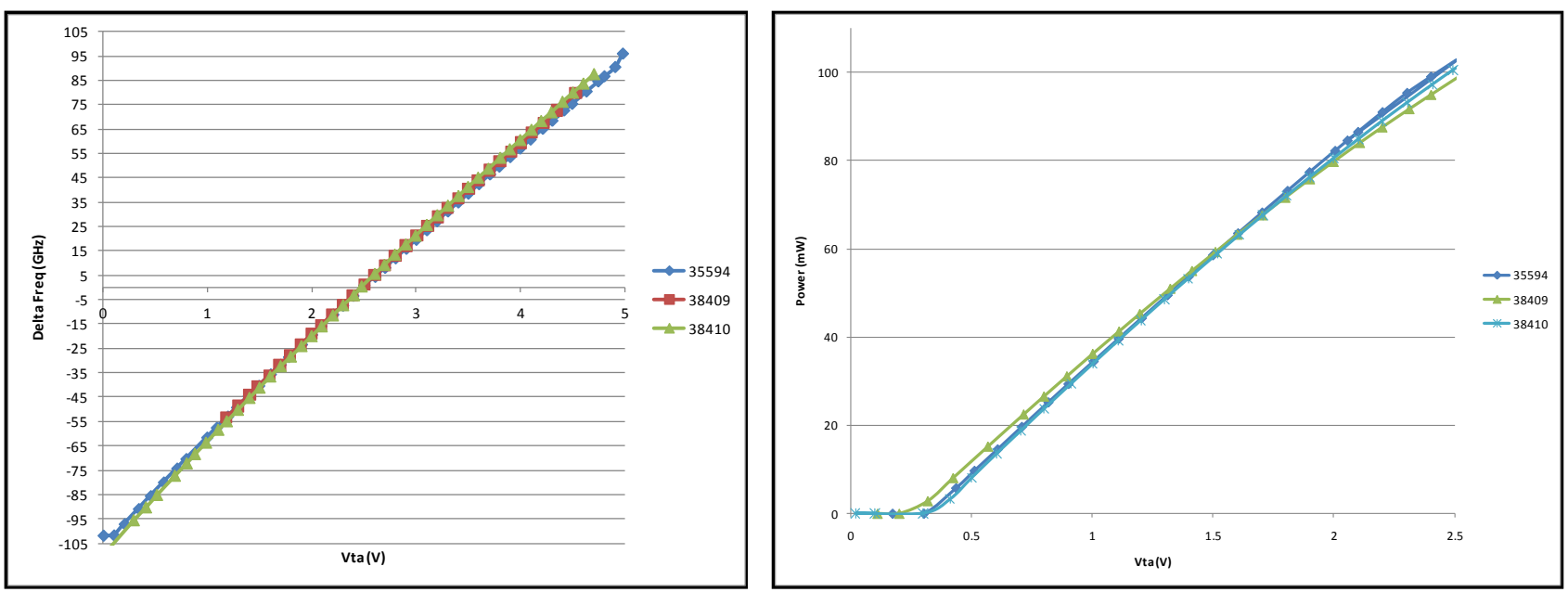

Figure 7. Frequency tuning characteristics. Frequency tuning via temperature adjust input for first three prototypes. Power adjust tuning curves for the first three prototype EM650s.

\section{LINEWIDTH AND NOISE MEASUREMENTS}

The linewidth of the EM650 was measured using the well-known delayed self-heterodyne technique. Frequency stability was measured by heterodyning two free-running laser modules. Frequency offset was adjusted to $\sim 1 \mathrm{GHz}$.

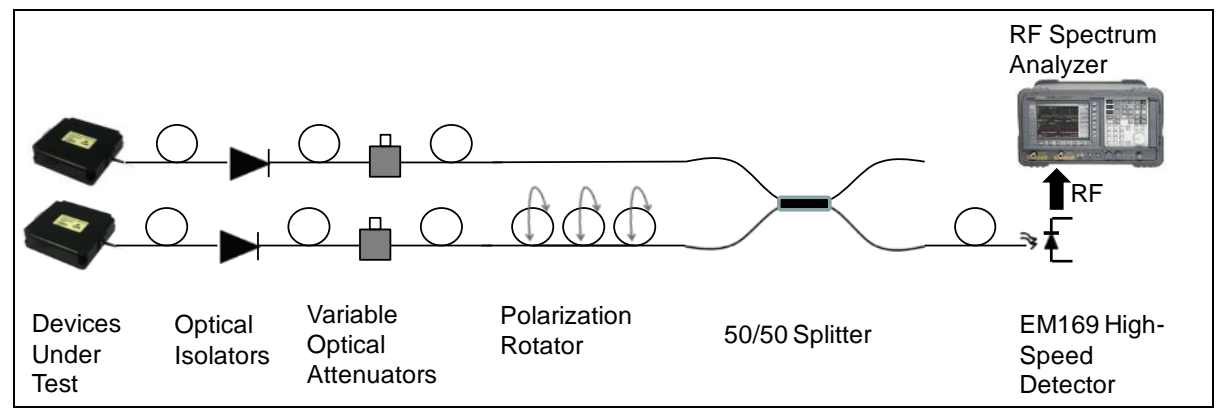

Figure 8. Schematic of the heterodyne measurement technique.

Commercial measurement systems have difficulty in measuring line stability $<\sim 200 \mathrm{MHz}(1.6 \mathrm{pm}$ at $\lambda=1550 \mathrm{~nm})$. The simple heterodyne technique that we have utilized provides a semi-quantitative measure of line positional stability and a qualitative comparison of linewidth. The measurement technique we are using is the standard Delayed Self-Heterodyne (DSH technique), which has been widely published [6-9] is illustrated in Figure 8. The details of the experimental setup are as follows. The laser output was attenuated and fed into a Mach-Zehnder interferometer with a very long delay. The input beam is first split into two legs using a fiber optic splitter/coupler. In one leg an $80 \mathrm{MHz}$ acousto-optic frequency shifter is employed to supply a difference frequency about which the linewidth spectrum can be measured. In the other leg four spools of Corning SMF-28e+ fiber with a total length of approximately $80 \mathrm{~km}$ are used to de-cohere the two beams. The delayed and frequency shifted beams are recombined using a second fiber optic splitter/coupler. The splitters, AO frequency shifter, and delay spools are housed in a vibration isolated enclosure to minimize the effects of laboratory acoustics on the linewidth measurement. The recombined optical signal generates a beat signal on an EM169 photodetector whose output is fed into an electrical spectrum analyzer. The beat signal about the $80 \mathrm{MHz}$ difference frequency is averaged for two seconds and fit to a pure Lorentzian curve. Figure 9 and Figure 10 illustrate linewidth measurements. The results for EM650 module indicate $\sim 3 \mathrm{x}$ better performance than currently available commercial-offthe-shelf drivers. The Lorentzian linewidths of $11 \mathrm{kHz}$ shown in Figure 10 are the full width $($ at $-3 \mathrm{~dB})$ of the measured 
beat-note spectra, and are therefore considered conservative estimates of the laser linewidth. Figure 11 illustrates relative intensity noise characteristics. The intensity noise is low even at low frequencies. In Figure 12, the measured phase noise spectrum with a noise corner around $20 \mathrm{kHz}$. This phase noise curve verifies that there are no peaks with the drive electronics that show up as phase noise. All we see is the typical $1 / \mathrm{f}$ curve transitioning to a flat white noise spectrum at higher frequencies.
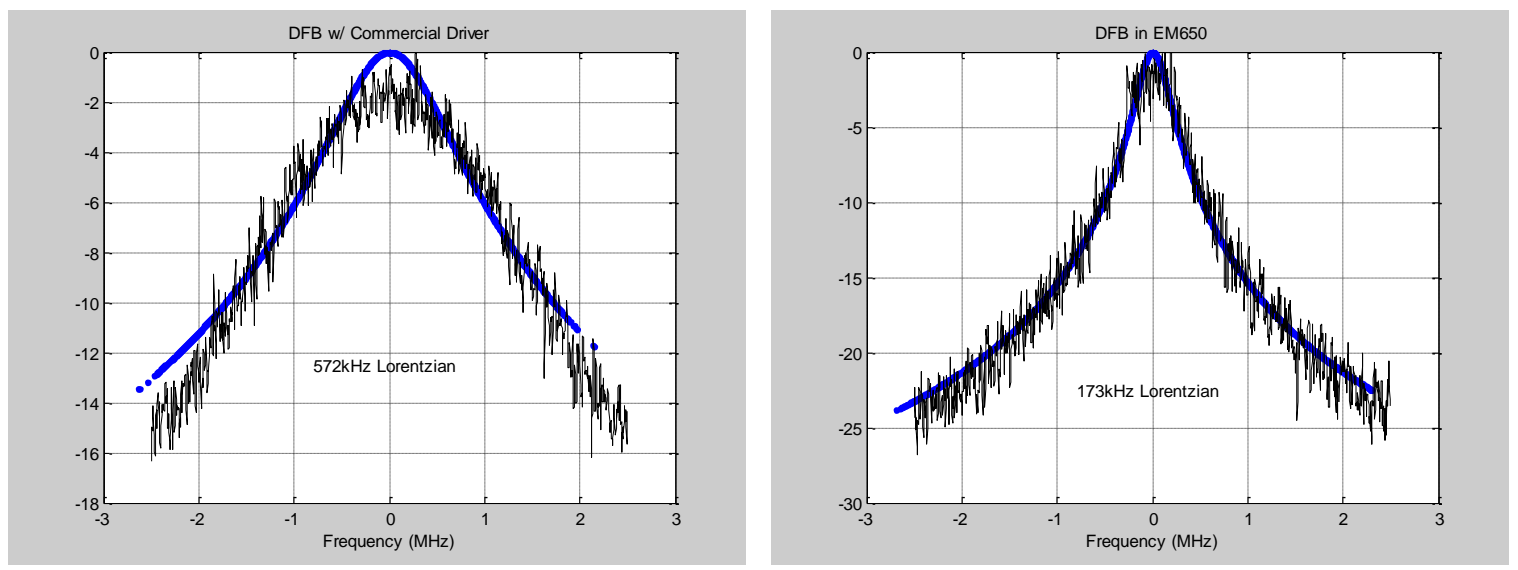

Figure 9. A DFB laser is driven to the same operating point using commercial benchtop equipment and a COTS EM650. The EM650 yield a linewidth $3 \mathrm{X}$ better than the lab driver

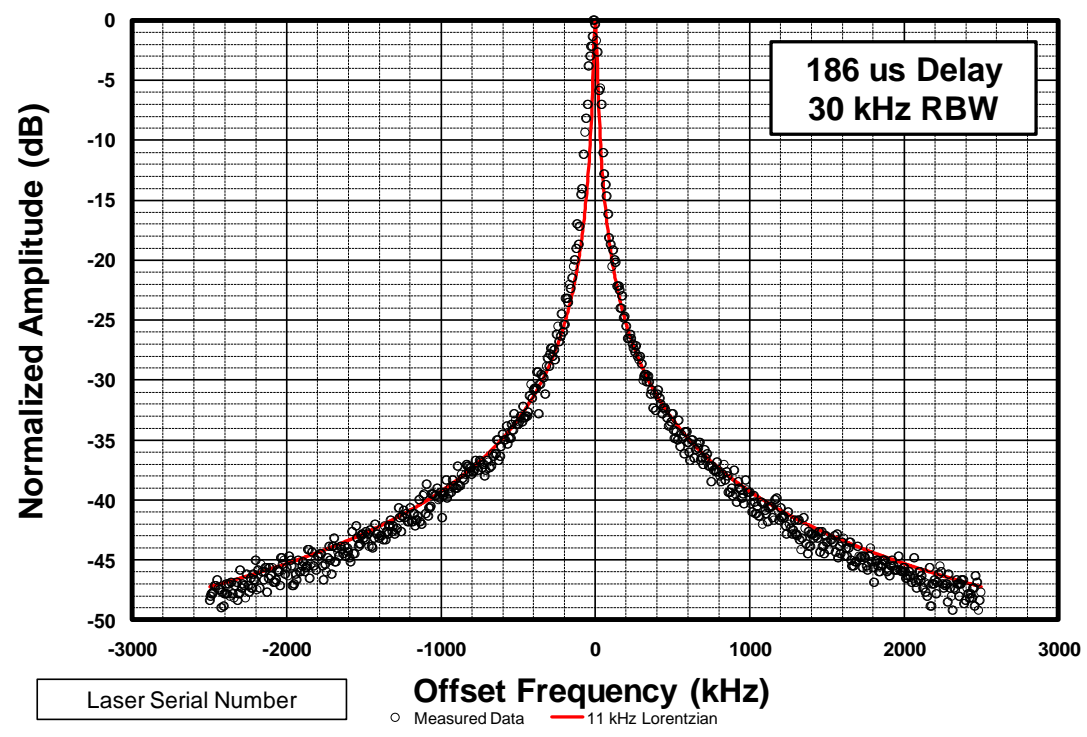

Figure 10. Typical linewidth measurements we have seen from an EM650, as measured by a national lab. Best performance observed is less than $11 \mathrm{kHz}$. 


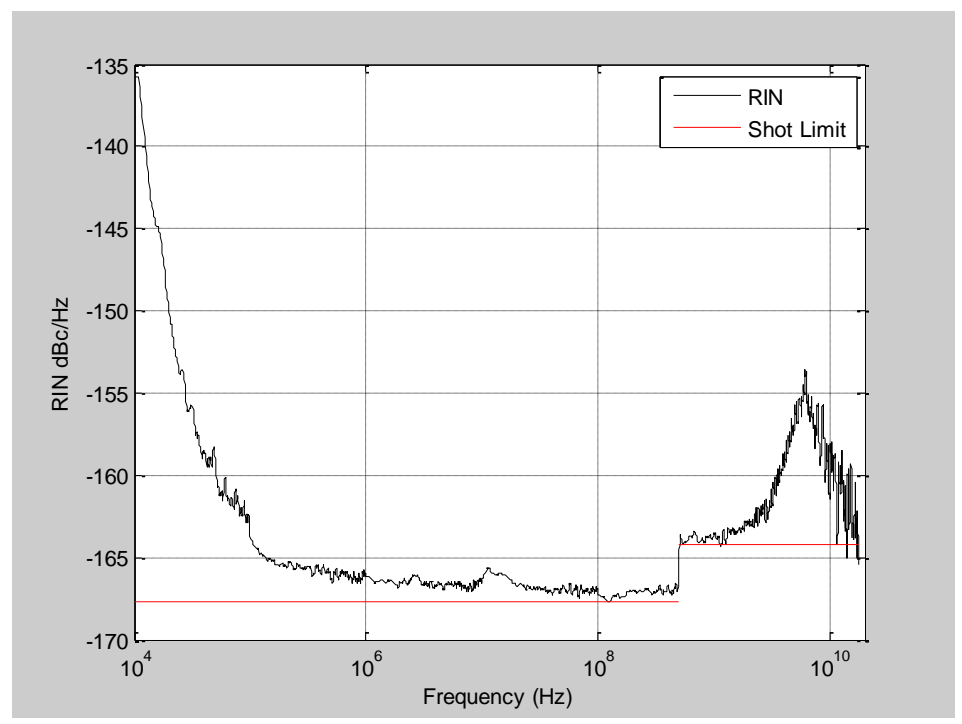

Figure 11. RIN demonstrated by 80mW EM650. Red lines are the detector shot noise limit for the measurement(s) shown in this graph. The intensity noise is low, even at low frequencies.

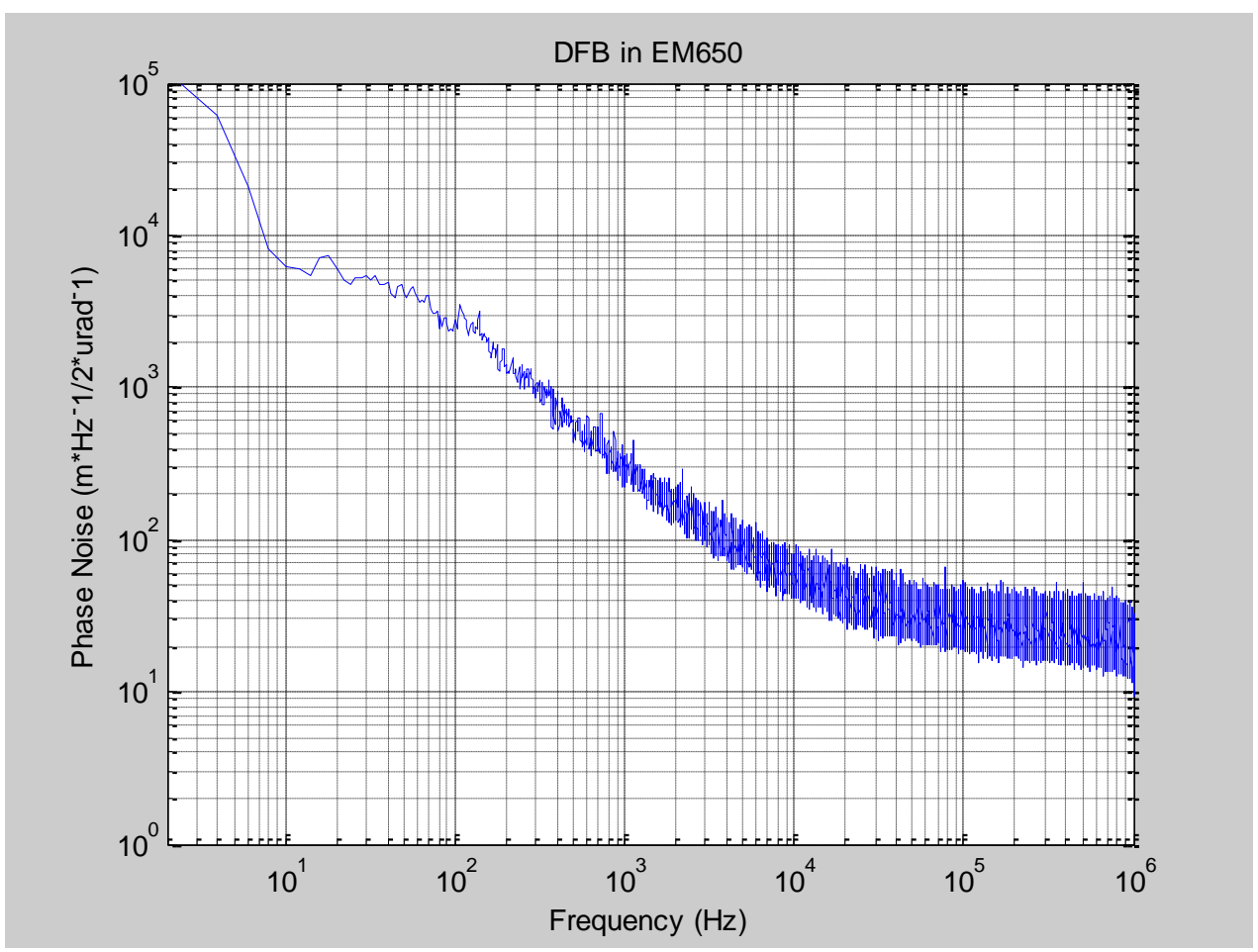

Figure 12. Phase noise curve of COTS EM650

\section{SUMMARY AND CONCLUSIONS}

An advanced, low SWaP seed laser transmitter with integrated control electronics for $\mathrm{CO}_{2}$ sensing applications for ASCENDS program has been built and tested. The new test devices (EM650) demonstrated enhanced coarse and fine wavelength tuning characteristics over previously developed module of EM600. With test devices operating at $1571 \mathrm{~nm}$, 
the tunability range of $\sim 2 \mathrm{~nm}$, with resolution of $0.2 \mathrm{pm} / \mathrm{mV}$, and stability of $0.8 \mathrm{pm}$ over 100 seconds has been achieved. Prototype devices for operation at 1260 spectral band are being fabricated. Lorentzian linewidth of $11 \mathrm{kHz}$ has been demonstrated. The measured RIN and phase noise curve characteristics are encouraging for space based high precision trace gas measurements. Preliminary tests have yielded promising results for LAS applications. Advanced nano-cooling scheme for reduction in power consumption as well as to improve linewidth and noise characteristics are being currently explored.

\section{REFERENCES}

1. NRC, Earth Science and Applications from Space: National Imperatives for the Next Decade and Beyond, The National Academies Press, Washington, D.C., 2007.

2. ASCENDS, NASA Science Definition and Planning Workshop Report, NASA ASCENDS Workshop, University of Michigan.

3. Narasimha S. Prasad, Alex Rosiewicz, Steven M. Coleman, "Development of a Low SWaP Laser Transmitter for Atmospheric Lidar Applications,” 2009 AGU Fall Meeting, San Francisco, CA, 14-18 December, 2009.

4. Narasimha S. Prasad, Alex Rosiewicz, Steven M. Coleman, "Development of a low SWaP laser transmitter for atmospheric lidar applications,” Proceedings of SPIE vol. 7912, San Francisco, CA, 22-27 January, 2011

5. http://www.em4inc.com/product_Integrated_Module.htm.

6. A. Yariv, "Quantum Electronics, $3^{\text {rd }}$ Ed." John Wiley \& Sons, pp. 596-597, 1989.

7. D. Dickerson, "Delayed Self-Heterodyne", in Fiber Optic Test and Measurement, New Jersey, Prentice Hall, pp. 185-188, 1998.

8. Y. Yamamoto, "Line Broadening in Semiconductor Lasers", Coherence, Amplification, and Quantum Effects in Semiconductor Lasers, New York, John Wiley \& Sons, pp. 22-23, 1991

9. http://www.rp-photonics.com/self_heterodyne_linewidth_measurement.html 